\title{
Transfering ultra-thin metallic metasurfaces on fibre endoscope probes for advanced imaging
}

\author{
Rafael Fuentes-Dominguez ${ }^{\mathrm{a}}$, Fei He ${ }^{\mathrm{a}}$, Richard Cousins ${ }^{\mathrm{b}}$, Christopher J. Mellor ${ }^{\mathrm{c}}$, and George S. \\ D. Gordon ${ }^{\mathrm{a}}$ \\ ${ }^{a}$ Optics and Photonics Group, University of Nottingham, University Park, Nottingham, NG7 \\ 2RD, UK \\ ${ }^{\mathrm{b} N a n o s c a l e}$ and Microscale Research Centre, University of Nottingham, University Park, \\ Nottingham, NG7 2RD, UK \\ ${ }^{\mathrm{c}}$ School of Physics and Astronomy, University of Nottingham, University Park, Nottingham, \\ NG7 2RD, UK
}

\begin{abstract}
In this paper we present a method to transfer ultra-thin polymer-encapsulated metallic metasurfaces onto optical fibers to enable ultra-thin imaging devices. The metasurface is first produced by conventional e-beam lithography on a silicon substrate and encapsulated by a resist layer. After patterning the resist layer to the target shape, the encapsulated metasurfaces are peeled off from the substrate and then glued onto the tip of a single- or multi-mode optical fiber. As a proof-of-concept we demonstrate a nanowire grating polarizer on the tip of an optical fibre. This method will allow the design and fabrication of multi-layered metasurface endoscopic devices for imaging and sensing.
\end{abstract}

Keywords: metallic metasurfaces, fiber optics, nanofabrications

\section{INTRODUCTION}

Imaging through hair-thin optical fibers has opened up new paradigms for biomedical imaging, such as in vivo bright/dark-field and fluorescence microscopy in living brains; ${ }^{1,2}$ quantitative phase/polarization imaging for early-stage cancer detection; ${ }^{3,4}$ and endoscopic confocal microscopy with high resolution. ${ }^{5}$ The state-of-art of this technology typically requires accurate characterisation of the transmission matrix (TM) of the light propagating through the fiber, ${ }^{6}$ which is inevitably sensitive to the ambient environment (i.e. mechanical deformation and/or temperature fluctuation) of living cells. Prior works have proposed different methods to perform real-time measurements of the TMs at the fiber distal facets, ${ }^{7-10}$ but with limited working range, strict preconditioning or large form factor. Gordon et al. ${ }^{11}$ proposed a novel way to characterise nonunitary fiber TMs without access to the distal facet meanwhile maintaining the hair-thin property of the fiber, which is by the introduction of a multilayer stack of spatially heterogeneous, partially reflecting metasurfaces and long-pass optical filters onto the distal facet.

Metasurfaces, which are ultrathin metamaterials, have provided flexible ways to control light by engineering the spatial distributions of amplitude, phase and polarization responses with subwavelength resolution. ${ }^{12-14}$ The majority of the readily demonstrated metasurfaces are top-down fabricated on millimeter-sized bulk substrates by electron-beam lithography (EBL), the configuration of which is not technologically compatible with the platform of fiber optics. Metasurfaces have previously been fabricated on optical fiber facets, via either direct milling into deposited layers or indirect transfer of predefined patterns with sacrificial substrates, ${ }^{15-17}$ but these methods are not well-suited to flexibly transfer multilayer stacks of custom-designed patterns onto fiber tips. Such designs are necessary to unlock the full range of possible fibre imaging devices.

Further author information: (Send correspondence to Rafael Fuentes-Dominguez)

R.F.D.: E-mail: rafael.fuentesdominguez1@nottingham.ac.uk, Telephone: +44 (0)115 9515605

G.S.D.G.: E-mail: george.gordon@nottingham.ac.uk 

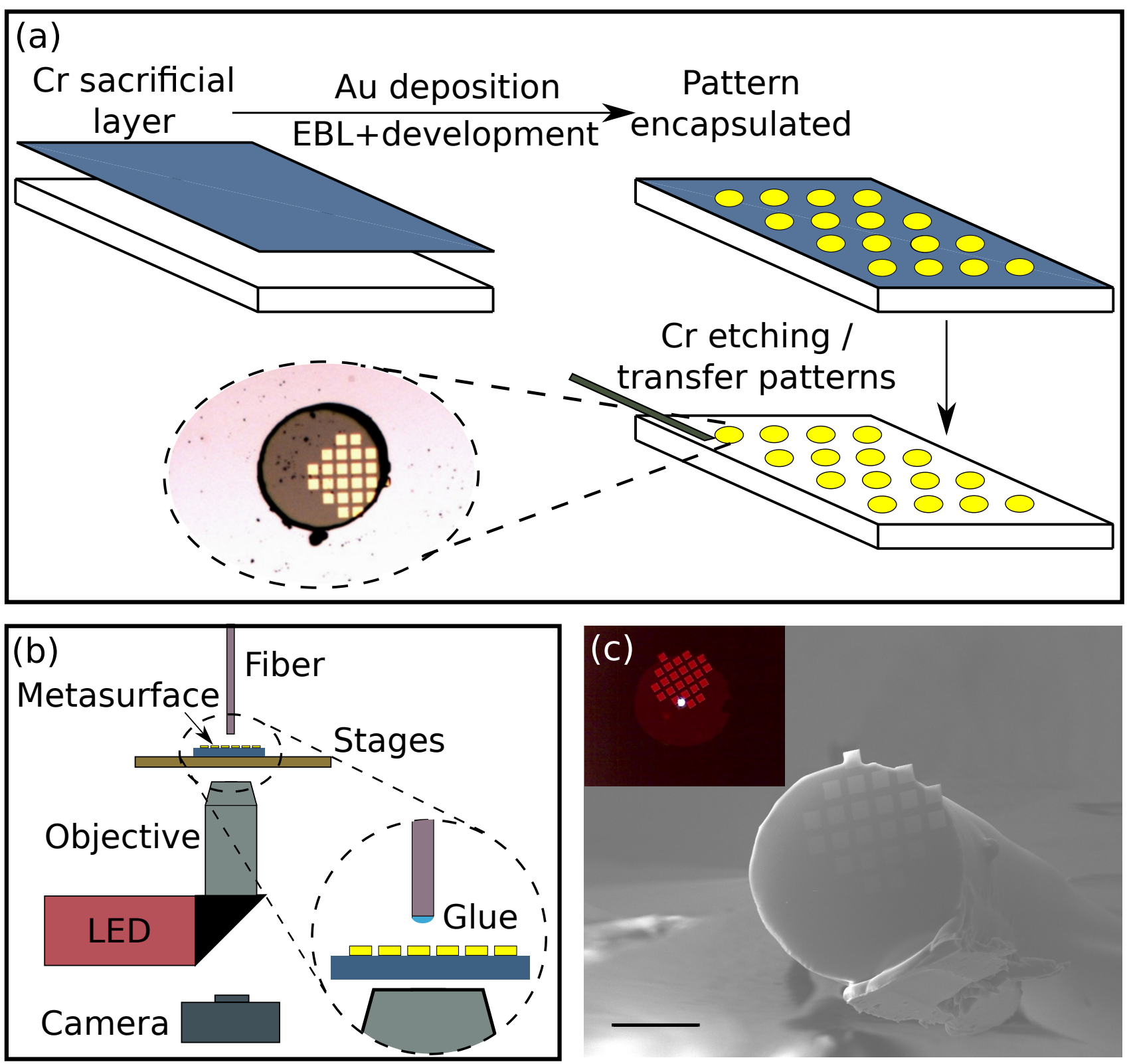

Figure 1. Ultra-thin polymer-encapsulated metallic metasurfaces fabrication showing the steps to encapsulate (a) and transfer them to an optical fiber (b). (c) Scanning electron microscopy image of a metallic metasurface stuck on the tip of a single-mode optical fiber (scale bar: $50 \mu \mathrm{m}$ ). Inset is the optical image through the experimental set-up with a white dot indicating the core of the single-mode fibre.

In this work, we show a new method to fabricate and transfer ultra-thin metallic metasurfaces onto the tips of optical fibers, which can be easily scaled up to fabricate multilayer metasurface stacks. The fabrication involves conventional EBL process to define metasurfaces on a silicon $(\mathrm{Si})$ substrate and encapsulation of the metasurfaces by a photo-resist layer (CAR44). After patterning the resist layer to the target shape, the encapsulated metasurfaces are transferred from the substrate and glued onto the tips of single- and/or multi-mode fibers. As a proof-of-principle, we fabricate and optically characterise a wire-grid polarizer metasurface on the tip of an optical fibre, which represents a novel approach to the generation of linear polarization states that is important in many advanced imaging applications..$^{41}$ 


\section{METHODOLOGY}

\subsection{Fabrication of polymer-encapsulated metallic metasurfaces}

The first step is to fabricate and encapsulate the desired metallic metasurfaces using the following process (shown in Figure 1(a)).

A silicon substrate is coated with a $30 \mathrm{~nm}$ sacrificial layer of chrome $(\mathrm{Cr})$. Then, the metallic metasurface is fabricated by conventional electron-beam lithography on the substrate. This includes spin-coating of resist, electron beam exposure, development, gold deposition (22 nm thick) and lift-off processes.

To encapsulate the metallic metasurfaces, a $10 \mu \mathrm{m}$ layer of CAR44 resist is coated and patterned to the required shape using photo-lithography (in this case, a disc of $125 \mu \mathrm{m}$, matching the fiber diameter).

The chrome layer is removed by an etching process, so the encapsulated discs drop onto the silicon substrate. Finally, water is used to float the discs off the substrate with the aid of physical force from a needle to prepare them for adhesion to the optical fibre.

\subsection{Transferring metasurfaces onto a fiber}

Once the metallic metasurfaces are encapsulated on the resist and free from the sacrificial metallic layer, these are ready to be glued onto the fiber. First, the pattern is transferred from the silicon substrate to a glass substrate which enables imaging through the substrate and locating both metasurfaces and fiber in the field of view.

This is shown in Figure 1(b), where the sample is illuminated with a red LED through a 5x objective, and is imaged in reflection by a camera. The metallic metasurface is moved by electromechanical stages with $1 \mu \mathrm{m}$ step-size, whereas the fiber is translated in $\mathrm{x}, \mathrm{y}$ and $\mathrm{z}$ coordinates with manual micrometers. The fiber is also coupled with a white light source to enable accurate location of the core.

Once the metasurface and fiber are aligned and centered, UV-cured glue is attached to the fiber tip and pressed against the metasurface. The surface tension allows the metasurface to stick onto the tip and the glue is cured with a UV torch.

An example of a metallic metasurface glued to the tip of a single mode fiber is shown in Figure 1(c). Here, an scanning electron microscopy (SEM) picture demonstrates that the metasurface is stuck onto the tip successfully without being damaged during the process. Following setting of the glue, the same metasurface can be seen in the figure inset, viewed through the optical microscope of Figure 1(b).

\subsection{Nanowire grating polarizer design}

When designing a nanowire grating polarizer working in transmission, one is interested in its ability to transmit the light polarized along its major axis (Ex polarization in Figure 2(a)), and to block the polarization along its minor axis (Ey) at the designed wavelength $(850 \mathrm{~nm})$. This often yields a high extinction ratio of the transmission values $(\mathrm{Tx} / \mathrm{Ty})$, which means that any light filtered by the nanowire grating polarizer will have a dominant linear polarization state along the major axis. Based on this concept, we optimised the design of a bi-periodic nanowire array (Figure 2(a)) with the parameters shown in Table 1. The total length and width of the polarizer are 21.6 $\mu \mathrm{m}$ and $150 \mathrm{~nm}$ respectively - the major axis is split into $3000 \mathrm{~nm}$ sections to aid fabrication.

We simulated the spectral dispersion of the nanowire transmissions with input polarizations along the major (Tx) and minor-axis (Ty) directions, as shown in Figure 2(b). The major-axis polarization gives a broadband high-level transmission, with $62 \%$ at the designed wavelength of $850 \mathrm{~nm}$ (blue solid line). On the other hand, the light polarized along the minor axis has a transmission valley of $1.4 \%$ at this wavelength (blue dashed line). According to the simulations, the high extinction ratio $(\mathrm{Tx} / \mathrm{Ty})$ of 44 at $850 \mathrm{~nm}$ (orange line) indicates that the designed nanowire grating polarizer should give a good performance at this wavelength.

Furthermore, we simulated the variations of the polarizer extinction ratio against two design parameters, nanowire width and minor-axis gap, as illustrated in Fig. 2. Within the ranges of parametric sweep, the extinction ratio is almost invariant to the minor-axis gap (thought it is more pronounced outside the simulated range), while it is highly sensitive to the change of the nanowire width. Given a fixed gap value (i.e. $110 \mathrm{~nm}$ ) along the minor axis, the full width at half maximum (FWHM) of the peak ratio appears at $10 \%$ variation of 

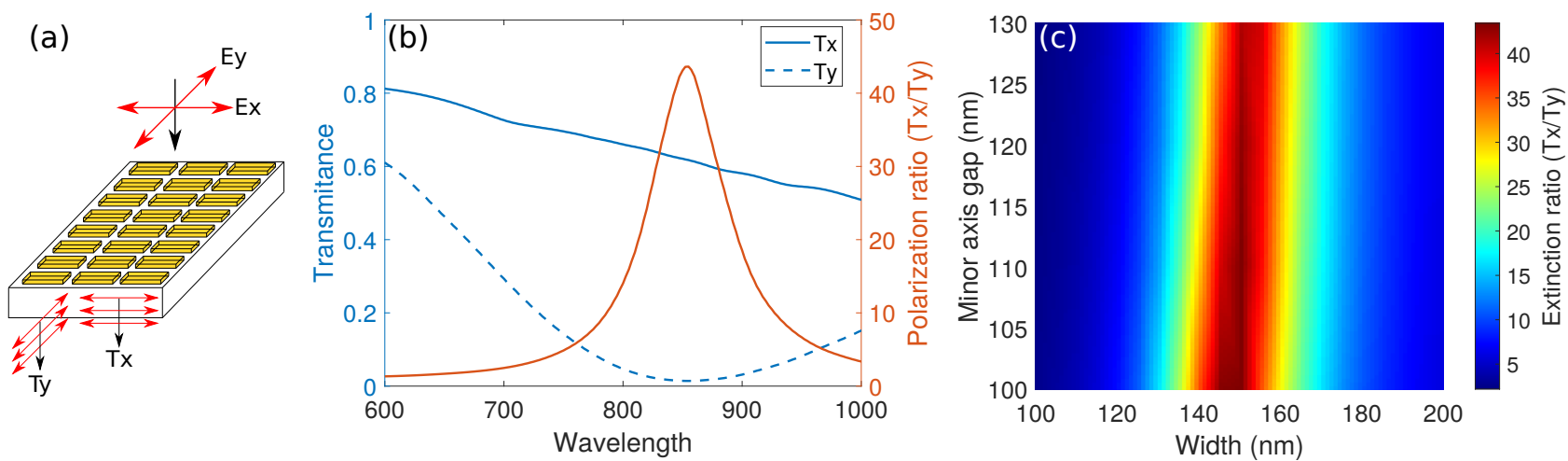

Figure 2. (a) Diagram of the nanowire grating polarizer and input / output light polarization. (b) Simulated transmittance spectra for a nanowire grating polarizer with parameters shown in Table 1 . The polarization ratio (Tx/Ty) is about 44 for $850 \mathrm{~nm}$ wavelength. (c) Extinction ratio map for different values of nanowire width and minor axis gap.

the width, and the ratio is even 10 times lower than the peak at $30 \%$ variation. This imposes tight tolerances for fabrication precision, as a slight variation in the polarizer dimension can significantly compromise its performance for the generation of linear polarization states.

Table 1. Design parameters of nanowire grating polarizer

\begin{tabular}{|c|c|}
\hline Parameter & Dimensions (nm) \\
\hline Length nanowire & 3000 \\
\hline Width nanowire & 150 \\
\hline Gap (along major axis) & 100 \\
\hline Gap (along minor axis) & 110 \\
\hline Thickness & 22 \\
\hline
\end{tabular}

\section{RESULTS}

After maximising the extinction ratio for multiple parameters, the nanowire grating polarizer design was fabricated using the steps given previously. Figure 3(a)-(b) show SEM images of the polarizers before being encapsulated on the resist, and figure 3(c) is the optical image after encapsulation.

After transferring the nanowire grating polarizer to a single mode fiber, the polarization behaviour was measured. To do this, linearly polarized light at different axis angles was generated using a fixed linear polarizer, followed by a quarter-wave plate followed by a rotating linear polariser. The resulting light was then coupled via the nanowire grating polarizer into the fibre measured with a power meter. The coupled power was measured as the polarisation angle of the incident light was swept from $0^{\circ}$ to $180^{\circ}$. This is shown in Figure $3(\mathrm{~d})$ where the maximum value is achieved when the input polarization matches the polarization axis of the nanowire grating polarizer. The measured extinction ratio obtained for this nanowire grating polarizer (see inset) is 2.37.

The difference between the simulated and experimental extinction ratio may be explained by fabrication tolerances. As shown in 2(c), small changes in the width and gap between nanowires can greatly reduce the polarizer performance. For instance, $10 \%$ variation in nanowire width can reduce the polarization ratio by half.

It is also important to notice that any defects on the polarizers smaller than the resolution limit of the optical setup cannot be imaged. This could explain the reduction of the extinction ratio of the nanowire grating polarizer if a defective sample is used.

Though there is clearly room for improvement of this specific design, it nonetheless serves as a proof-ofprinciple that this process is feasible, flexible and scalable to multi-layer stacks of metasurfaces. 

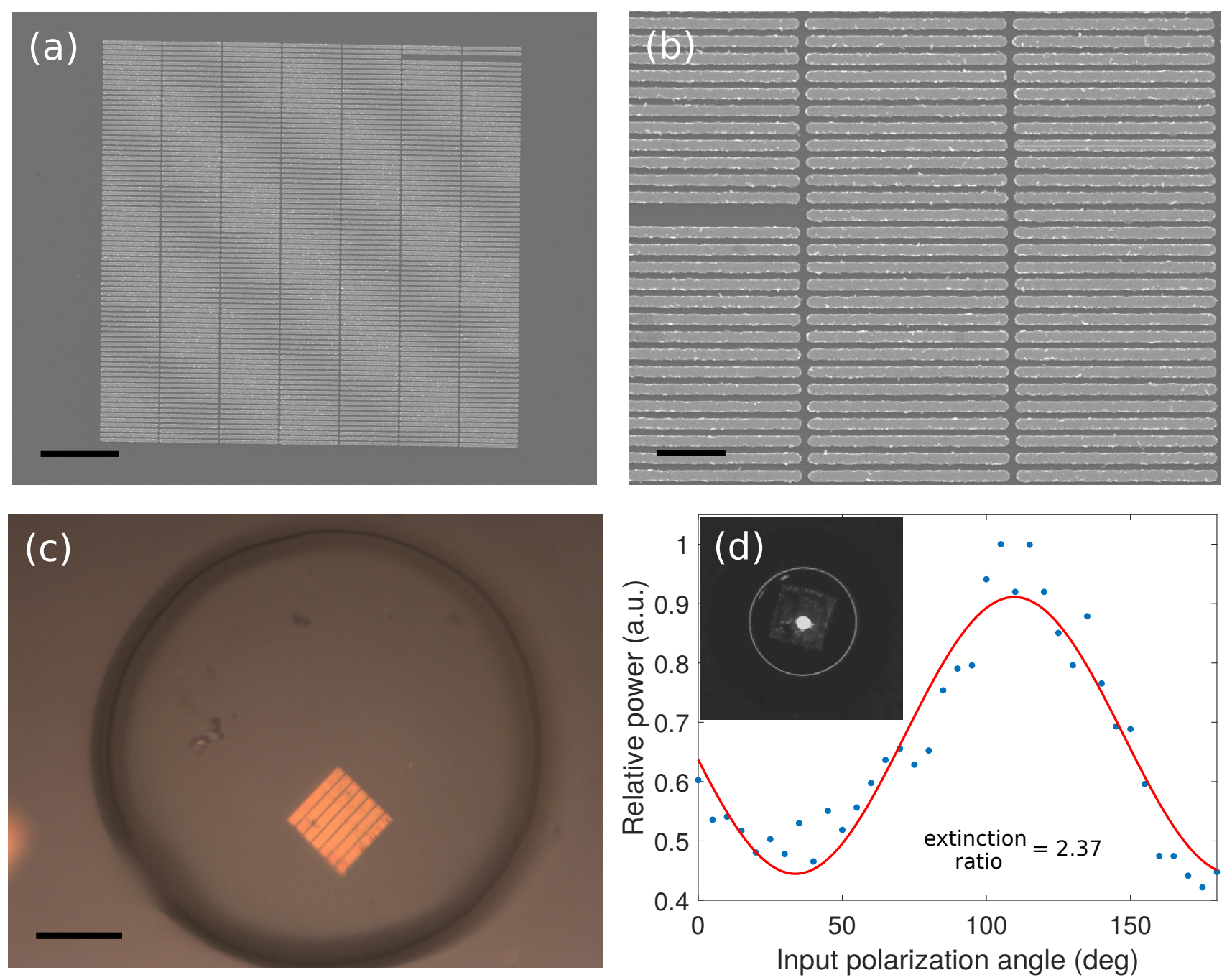

Figure 3. Nanowire grating polarizer. (a) Scanning electron microscopy (SEM) image of the polarizer before being encapsulated (scale bar: $3 \mu \mathrm{m}$ ). SEM zoomed area (scale bar: $1 \mu \mathrm{m}$ ). The design parameters are shown in Table 1. (c) Optical microscopy image of the nanowire grating polarizer encapsulated on the resist, CAR44, with $125 \mu \mathrm{m}$ diameter (scale bar: $25 \mu \mathrm{m}$ ). (d) The polarization performance of the nanowire grating polarizer on the tip of a single-mode optical fiber. The extinction ratio is about 2.37. The figure inset is the optical picture of the metasurface stuck on the fiber with the core illuminated.

\section{CONCLUSIONS}

Here, we have shown a method to transfer ultra-thin polymer-encapsulated metallic metasurfaces onto optical fibers. This method consists of fabricating metasurfaces by conventional e-beam lithography and encapsulating them in a resist layer (CAR44). After patterning the resist layer to the target shape, the encapsulated metasurface can be glued to the tip of single- or multi-mode optical fibers. As a proof of principle we have designed, fabricated, transferred and optically characterized a nanowire grating polarizer on an optical fiber.

\section{ACKNOWLEDGMENTS}

This work was supported by a UKRI Future Leaders Fellowship (MR/T041951/1) and a UNICAS Grant from the University of Nottingham. The authors would like to acknowledge the Nanoscale and Microscale Research Centre (nmRC) at the University of Nottingham for providing access to instrumentation and SEM imaging. 


\section{REFERENCES}

[1] Čižmár, T. and Dholakia, K., "Exploiting multimode waveguides for pure fibre-based imaging," Nature communications 3(1), 1-9 (2012).

[2] Vasquez-Lopez, S. A., Turcotte, R., Koren, V., Plöschner, M., Padamsey, Z., Booth, M. J., Čižmár, T., and Emptage, N. J., "Subcellular spatial resolution achieved for deep-brain imaging in vivo using a minimally invasive multimode fiber," Light: Science \&3 Applications 7(1), 1-6 (2018).

[3] Gataric, M., Gordon, G. S., Renna, F., Ramos, A. G. C., Alcolea, M. P., and Bohndiek, S. E., "Reconstruction of optical vector-fields with applications in endoscopic imaging," IEEE transactions on medical imaging 38(4), 955-967 (2018).

[4] Gordon, G. S., Joseph, J., Sawyer, T., Macfaden, A. J., Williams, C., Wilkinson, T. D., and Bohndiek, S. E., "Full-field quantitative phase and polarisation-resolved imaging through an optical fibre bundle," Optics express 27(17), 23929-23947 (2019).

[5] Loterie, D., Farahi, S., Papadopoulos, I., Goy, A., Psaltis, D., and Moser, C., "Digital confocal microscopy through a multimode fiber," Optics express 23(18), 23845-23858 (2015).

[6] Rotter, S. and Gigan, S., "Light fields in complex media: Mesoscopic scattering meets wave control," Reviews of Modern Physics 89(1), 015005 (2017).

[7] Farahi, S., Ziegler, D., Papadopoulos, I. N., Psaltis, D., and Moser, C., "Dynamic bending compensation while focusing through a multimode fiber," Optics express 21(19), 22504-22514 (2013).

[8] Plöschner, M., Tyc, T., and Čižmár, T., "Seeing through chaos in multimode fibres," Nature Photonics 9(8), $529-535$ (2015).

[9] Chen, H., Fontaine, N. K., Ryf, R., Neilson, D. T., Winzer, P., Gross, S., Riesen, N., Withford, M., $\mathrm{Li}$, J., Alvarado, J., et al., "Remote mode-forming over multimode fiber employing single-ended channel estimation," in [2018 European Conference on Optical Communication (ECOC)], 1-3, IEEE (2018).

[10] Gu, R. Y., Mahalati, R. N., and Kahn, J. M., "Design of flexible multi-mode fiber endoscope," Optics express 23(21), 26905-26918 (2015).

[11] Gordon, G. S., Gataric, M., Ramos, A. G. C., Mouthaan, R., Williams, C., Yoon, J., Wilkinson, T. D., and Bohndiek, S. E., "Characterizing optical fiber transmission matrices using metasurface reflector stacks for lensless imaging without distal access," Physical Review X 9(4), 041050 (2019).

[12] Chen, H.-T., Taylor, A. J., and Yu, N., "A review of metasurfaces: physics and applications," Reports on progress in physics $\mathbf{7 9}(7)$, 076401 (2016).

[13] Glybovski, S. B., Tretyakov, S. A., Belov, P. A., Kivshar, Y. S., and Simovski, C. R., "Metasurfaces: From microwaves to visible," Physics reports 634, 1-72 (2016).

[14] Kamali, S. M., Arbabi, E., Arbabi, A., and Faraon, A., "A review of dielectric optical metasurfaces for wavefront control," Nanophotonics 7(6), 1041-1068 (2018).

[15] Principe, M., Consales, M., Micco, A., Crescitelli, A., Castaldi, G., Esposito, E., La Ferrara, V., Cutolo, A., Galdi, V., and Cusano, A., "Optical fiber meta-tips," Light: Science 83 Applications 6(3), e16226-e16226 (2017).

[16] Flannery, J., Al Maruf, R., Yoon, T., and Bajcsy, M., "Fabry-pérot cavity formed with dielectric metasurfaces in a hollow-core fiber," ACS Photonics 5(2), 337-341 (2018).

[17] Yu, N. and Capasso, F., "Optical metasurfaces and prospect of their applications including fiber optics," Journal of Lightwave Technology 33(12), 2344-2358 (2015). 